\title{
Cell type-specific filamin complex regulation by a novel class of HECT ubiquitin ligase is required for normal cell motility and patterning
}

\author{
Simone L. Blagg ${ }^{1}$, Suzanne E. Battom ${ }^{1}$, Sarah J. Annesley ${ }^{2}$, Thomas Keller ${ }^{1}$, Katie Parkinson ${ }^{1}$, \\ Jasmine M. F. Wu ${ }^{1}$, Paul R. Fisher ${ }^{2}$ and Christopher R. L. Thompson ${ }^{1, *}$
}

\begin{abstract}
SUMMARY
Differential cell motility, which plays a key role in many developmental processes, is perhaps most evident in examples of pattern formation in which the different cell types arise intermingled before sorting out into discrete tissues. This is thought to require heterogeneities in responsiveness to differentiation-inducing signals that result in the activation of cell type-specific genes and 'salt and pepper' patterning. How differential gene expression results in cell sorting is poorly defined. Here we describe a novel gene $(h f n A)$ that provides the first mechanistic link between cell signalling, differential gene expression and cell type-specific sorting in Dictyostelium. HfnA defines a novel group of evolutionarily conserved HECT ubiquitin ligases with an N-terminal filamin domain (HFNs). HfnA expression is induced by the stalk differentiation-inducing factor DIF-1 and is restricted to a subset of prestalk cells (pstO). $h f n A^{-}$pstO cells differentiate but their sorting out is delayed. Genetic interactions suggest that this is due to misregulation of filamin complex activity. Overexpression of filamin complex members phenocopies the $h f n A^{-}$pstO cell sorting defect, whereas disruption of filamin complex function in a wild-type background results in pstO cells sorting more strongly. Filamin disruption in an $h f n A^{-}$background rescues pstO cell localisation. $h f n A^{-}$cells exhibit altered slug phototaxis phenotypes consistent with filamin complex hyperactivity. We propose that HfnA regulates filamin complex activity and cell type-specific motility through the breakdown of filamin complexes. These findings provide a novel mechanism for filamin regulation and demonstrate that filamin is a crucial mechanistic link between responses to differentiation signals and cell movement in patterning based on 'salt and pepper' differentiation and sorting out.
\end{abstract}

KEY WORDS: Cell sorting, Dictyostelium, Filamin, Patterning

\section{INTRODUCTION}

Multicellular development requires the differentiation of different cell types and their correct positioning within the embryo. In most organisms, this developmental patterning process requires some cells to move from their site of differentiation to different positions. For directed cell movement to occur, cells must be able to sense their position and orient accordingly, organise their motile machinery and maintain appropriate contacts with neighbouring cells. When these processes fail it can lead to severe developmental disorders and disease. Therefore, understanding how cell movements are coordinated within a three-dimensional multicellular environment represents one of the major challenges in developmental biology.

Cell movements are fundamental throughout development and are known to underpin processes such as gastrulation and the formation of the nervous system. One less-studied example, however, is illustrated by a mode of pattern formation in which different cell types differentiate randomly intermingled with each other, but then subsequently move and sort out to form discrete patterns. Cell sorting has been the subject of much research since it was demonstrated that dissociated cells from amphibian embryos

${ }^{1}$ Faculty of Life Sciences, Michael Smith Building, University of Manchester, Oxford
Road, Manchester M13 9PT, UK. ${ }^{2}$ Department of Microbiology, La Trobe University, Road, Manchester M1
VIC 3086, Australia

*Author for correspondence (christopher.thompson@manchester.ac.uk)

Accepted 25 January 2011 adhere to form random aggregates that subsequently sort out according to the germ layer of their origin (Townes and Holtfreter, 1955). However, it is now widely recognised that a similar mechanism also operates during normal development and is broadly evolutionarily conserved (Kay and Thompson, 2009). For example, during the earliest stages of mouse embryonic development the inner cell mass is divided into the pluripotent epiblast, which gives rise to the embryo proper, and the primitive endoderm, which forms the yolk sac. Prior to the segregation of these lineages, the precursors can already be detected by the expression of lineage-specific transcription factors. However, rather than the distribution of the precursor cells being position dependent, they show a 'salt and pepper' distribution. These two different cell types subsequently sort out into their appropriate layers by the late blastocyst stage (Chazaud et al., 2006; Chisholm and Houliston, 1987; Dietrich and Hiiragi, 2007; Kurimoto et al., 2006; Plusa et al., 2008).

Perhaps the best illustration of patterning by sorting out is during the developmental cycle of the social amoeba Dictyostelium discoideum. Dictyostelium development is triggered by the onset of starvation, resulting in the aggregation of separate amoebae in response to extracellular cAMP, which acts as a chemoattractant (Kessin, 2001). The resulting 'mound' then develops into the motile 'slug' and finally, when conditions are favourable, a 'fruiting body' is formed. This terminally differentiated structure consists of spore cells, which are alive and are dispersed into the soil, and stalk cells, which are dead and function to hold aloft the spore cells (Kessin, 2001). The prestalk cells can be further subdivided into prestalk A cells (pstA, found at the very tip of the slug), prestalk 
$\mathrm{AB}$ cells (pstAB, a cone of cells within the A region), prestalk $\mathrm{B}$ cells (enriched at the prestalk/prespore boundary and dotted around the prespore region) and prestalk $\mathrm{O}$ cells (pstO, a collar of cells behind the pstA region) (Williams, 2006). A number of signalling molecules are essential for proper cell type specification. The most studied of these is differentiation-inducing factor 1 (DIF-1) (Kay, 1998), which is essential for the specification of pstO and pstB cells (Keller and Thompson, 2008; Saito et al., 2008; Thompson and Kay, 2000). DIF-1 is known to affect gene transcription in prestalk cells via the activation of a number of transcription factors, including STATc (Fukuzawa et al., 2003), DimA (Thompson et al., 2004a), DimB (Huang et al., 2006; Zhukovskaya et al., 2006) and GATAc (Keller and Thompson, 2008).

It is now generally accepted that the different cell types are initially specified intermingled, without positional information, followed by subsequent cell sorting and pattern formation (Kay and Thompson, 2009; Thompson et al., 2004a; Williams et al., 1989a). For example, the cells destined to become stalk cells (prestalk cells) first differentiate in the mound, intermingled with prespore cells, and then sort to the front of the slug, whereas the prespore cells are found in the rear of the slug (Williams et al., 1989b). Furthermore, if cell movement is blocked at the mound stage, when differentiation begins, the cell types remain intermingled and no pattern is formed (Kay and Thompson, 2009; Thompson et al., 2004b). Different models have been proposed to explain how differential motility and cell sorting are regulated in Dictyostelium and other organisms. For example, it has been suggested that cells could sort due to their specific adhesive properties or surface tension (Clow et al., 2000; Jiang et al., 1998; Siu et al., 2004). Furthermore, there is good evidence that differential chemotactic cell motility may play an important role. Indeed, Dictyostelium prestalk and prespore cells exhibit different speeds of movement in response to a cAMP stimulus (Early et al., 1995; Lam et al., 1981; Matsukuma and Durston, 1979; Siegert and Weijer, 1995; Traynor et al., 1992).

Consequently, it has been proposed that the sorting of prestalk cells is likely to require the coordination of differentiation, motility and adhesion. As DIF-1 regulates pstO cell differentiation, we therefore reasoned that DIF-1 targets would represent candidate genes for pstO cell sorting. However, relatively few DIF-1regulated genes have been identified (Maruo et al., 2004). Furthermore, none of these has been shown to be required for cell fate choice or sorting (Morrison et al., 1994). It is of vital importance to identify downstream DIF-1 targets to elucidate the mechanisms that drive patterning. We have found that Dictyostelium HfnA is induced by DIF and is expressed in pstO cells. The $h f n A$ gene encodes a protein with significant sequence homology to both HECT ubiquitin ligases and filamin.

HECT domain-containing ubiquitin ligases target proteins for destruction via the proteasome. They play widespread biological roles and have been extensively linked to disease states in humans (Scheffner and Staub, 2007). However, relatively few of their targets have been identified (Scheffner and Staub, 2007). HECT ligases typically consist of a C-terminal HECT domain and a variable N-terminal domain (Huibregtse et al., 1995). The HECT domain is responsible for the addition of ubiquitin subunits to specific proteins (Scheffner et al., 1995), whereas the N-terminal domain is thought to confer target specificity by binding to its particular substrate (Scheffner and Staub, 2007). As HfnA contains N-terminal sequences similar to those found in filamin, we hypothesised that HfnA might be involved in filamin complex regulation during Dictyostelium development.
Filamins are F-actin-binding proteins that are thought to function as adaptor proteins, bringing together elements of the cytoskeleton and signal transduction proteins (Bandala-Sanchez et al., 2006; Popowicz et al., 2006). Filamins contain actin-binding domains at their N-termini, followed by several filamin repeats. Each filamin repeat is $\sim 100$ amino acids long and contains an immunoglobulin fold (Noegel et al., 1989). The repeats function as binding domains, through which filamins can dimerise (Fucini et al., 1999). Over 30 diverse protein interactions with vertebrate filamins have been identified, which include transcription factors such as FOXC (Berry et al., 2005), many cytoskeletal and adhesion proteins such as migfilin (Tu et al., 2003), GTPases (Ohta et al., 1999), kinases and receptors (Popowicz et al., 2006). Unsurprisingly for a protein with such complex interactions, filamins are associated with a number of disease states and developmental disorders. In humans, there are three filamins (A, B and C), with filamin A (FLNA) being the most widely studied. Loss-of-function mutations in FLNA lead to periventricular nodular heterotopia, a developmental disorder that results from defective neuronal migration (Fox et al., 1998). However, other mutations that alter filamin function also result in a large number of different developmental malformations, indicating that the precise regulation of filamin is essential (Robertson, 2005). Filamin has multiple modes of regulation, including phosphorylation and degradation by calpain (Chen and Stracher, 1989); although regulation by ubiquitylation has not been reported, the identification of an evolutionarily conserved filamin domaincontaining HECT ligase has suggested that ubiquitylation could play a vital role in filamin complex regulation.

Dictyostelium contains one filamin (Fln) homologue, which consists of an actin-binding domain at its N-terminus followed by six filamin repeat domains. Filamin functions as a dimer, and dimerisation requires repeats 5 and 6 (Fucini et al., 1999). In Dictyostelium, filamin has been shown to play roles in the organisation of the actin cytoskeleton, in pseudopod formation and in the speed of movement of single cells. During development, however, the major role of filamin appears to be in slug phototaxis (Fisher et al., 1997; Khaire et al., 2007), which requires repeats 26 (Annesley et al., 2007). Filamin has been shown to function in the tip of the slug, in the pstA region, as expression of full-length filamin under the control of a pstA-specific promoter is able to completely rescue the Fln-null phototaxis phenotype (Khaire et al., 2007). Filamin has been proposed to act as an adaptor protein at the front of the slug during this process, as it is found within a large signalling complex that comprises many proteins, including RasD, ERKB, GRP125 and PKB (Bandala-Sanchez et al., 2006). Whereas numerous binding partners of filamin have been identified in mammalian cells (Popowicz et al., 2006), relatively few have been identified in Dictyostelium. The best characterised is filamininteracting protein (FIP), which is developmentally regulated, whereas filamin is not (Knuth et al., 2004). FIP has been demonstrated to interact with filamin in vivo and is required for the correct regulation of filamin (Knuth et al., 2004). FIP colocalises with filamin in Dictyostelium cells and has been shown to directly bind to filamin between repeats 2 and 4 (Knuth et al., 2004). Although attempts to make an FIP-null mutant have been unsuccessful (Knuth et al., 2004), overexpression of a C-terminal fragment of FIP (cFIP) has been reported to cause defects consistent with a role in filamin regulation (Knuth et al., 2004).

We demonstrate here that the DIF-1-induced, filamin domaincontaining HECT ubiquitin ligase HfnA affects filamin complex activity. Furthermore, we show that $\mathrm{HfnA}$ is necessary for normal development and correct pstO cell motility in a three-dimensional 
environment during pattern formation. These findings suggest that differential HfnA expression and filamin complex activity provide a key regulatory step in the mechanism by which heterogeneous salt-and-pepper gene expression can lead to organised tissue patterning.

\section{MATERIALS AND METHODS}

\section{Cell culture and development}

Dictyostelium discoideum strains were grown in axenic medium (HL5) (Sussman, 1987) or on Klebsiella lawns. For development, axenically growing cells were washed in $\mathrm{KK} 2\left(16.1 \mathrm{mM} \mathrm{KH}_{2} \mathrm{PO}_{4}, 3.7 \mathrm{mM} \mathrm{K}_{2} \mathrm{HPO}_{4}\right)$ and plated at $2 \times 10^{7}$ cells per $10-\mathrm{cm} 1.5 \% \mathrm{KK} 2$ agar plate and left in the dark. For analysis of Dictyostelium plaque 'slugging', Dictyostelium cells were spotted onto Klebsiella lawns on SM agar plates and left in a humid chamber for 5 days.

\section{Microarray experiment}

Microarray experiments were performed as described (Van Driessche et al., 2002). Each sample was analysed by a two-colour assay in which test samples were compared with a common pooled reference sample of total RNA from several wild-type developmental time points. The samples were hybridised to a microarray of 5669 unique targets. A single-chip normalisation procedure was used to allow for multi-array comparisons. Each hybridisation target was printed twice on the array, allowing for single-chip normalisation. At least two hybridisation experiments were carried out for each RNA extraction. Two biological replications were also performed. Technical and biological replicates were averaged to generate normalised timecourse data (Katoh et al., 2004; Van Driessche et al., 2002).

\section{Generation of $h f n A^{-}$and $h f n A^{-} / F I n^{-}$mutants}

Primers flanking the upstream and downstream regions of $h f n A\left(5^{\prime}\right.$ GATGAGCGGGGAGAATTCTTG-3' and 5'-AGAAACCATCTCCTTCAGTGA-3') were used to generate a $3.5 \mathrm{~kb}$ genomic fragment that was cloned into TOPO TA 2.1 vector (Invitrogen). A gene-disruption construct was generated by in vitro transposition (Abe et al., 2003) of a blasticidin resistance cassette into the HECT domain. This construct was introduced into wild-type AX4 cells by electroporation and mutants were selected at $10 \mu \mathrm{g} / \mathrm{ml}$ blasticidin. Blasticidin-resistant colonies were screened by PCR for homologous recombination.

The filamin gene $a b p C$ was disrupted in the $h f n A^{-}$background using hygromycin as a selectable marker (Egelhoff et al., 1989). The C-terminal FIP fragment expression construct (Knuth et al., 2004) was introduced into cells by electroporation and selected at $10 \mu \mathrm{g} / \mathrm{ml} \mathrm{G} 418$.

\section{lacZ marker studies}

A $0.9 \mathrm{~kb}$ fragment upstream of the $h f n A$ transcription start site was cloned into vector pDGal17 (Harwood and Drury, 1990). This construct and ecmO-lacZ, ecmA-lacZ and ecmAO-lacZ were electroporated into AX4 and mutant cells and the resultant transformants selected at $10 \mu \mathrm{g} / \mathrm{ml} \mathrm{G} 418$. lac $Z$ whole-mount staining was then performed as previously described (Dingermann et al., 1989) as well as measurements of $\beta$-galactosidase activity upon DIF-1 induction in monolayer culture, as described (Thompson et al., 2004a). For each experiment, several thousand transformants were pooled and the result confirmed with duplicate electroporations

\section{Analysis of cell movement}

For analysis of movement during multicellular development, transformants were developed to the mound stage. A thin strip of agar was cut out and transferred to a humid chamber on its side to view structures 'side on'. Cell tracking was performed using ImageJ software and the Manual Tracking plug-in. To measure chemotaxis of dissociated mound stage cells, mounds were transferred to KK2 medium containing $0.1 \%$ pronase, and dissociated by gently syringing through a $20 \mathrm{G}$ needle. Then $2-5 \times 10^{4}$ cells were plated onto glass-bottomed dishes (World Precision Instruments) and allowed to settle for 30 minutes. Cells were then stimulated with a micropipette filled with KK2 containing $2 \mathrm{mM} \mathrm{MgSO}_{4}$ and $100 \mu \mathrm{M}$ cAMP. Centroid analysis was performed using ImageJ software. In addition to cell speed, chemotaxis index and persistence were calculated. Persistence was calculated from the cosine of the angle between two consecutive movement steps. Chemotaxis index was determined as the ratio of net cell displacement over the course of the movie and total distance travelled.

\section{Phototaxis}

Qualitative phototaxis tests were performed as described (Darcy et al., 1994). A toothpick scraping of amoebae was transferred from a colony growing on a Klebsiella lawn to the centre of charcoal agar plates $(5 \%$ activated charcoal, $1 \%$ agar). The plates were incubated for 48 hours at $21^{\circ} \mathrm{C}$ with a lateral light source and the phototaxis was scored. For quantitative phototaxis tests, amoebae were harvested from mass plates, thoroughly washed free of bacteria, and suspended in saline at the appropriate dilutions. Then $20 \mu 1$ of the amoebal dilutions were inoculated onto a $1 \mathrm{~cm}^{2}$ area in the centre of each charcoal agar plate. The resulting cell densities ranged from $\sim 2 \times 10^{6}$ to $2.5 \times 10^{7}$ cells $/ \mathrm{cm}^{2}$. Phototaxis was again scored after 48 hours incubation at $21^{\circ} \mathrm{C}$ with a lateral light source. For both the qualitative and quantitative assays, slug trails were transferred to PVC discs and stained with Coomassie Blue before being digitised. Qualitative phototaxis test results are presented as complete, digitised trails plotted from a common origin. For statistical analysis of quantitative phototaxis experiments, the start and end points of slug trails were digitised and the orientation was analysed using directional statistics (P. R. Fisher, $\mathrm{PhD}$ thesis, Australian National University, 1981) (Fisher and Annesley, 2006).

\section{RESULTS A novel group of filamin domain-containing HECT ligases}

The Dictyostelium hfnA gene was identified as a DIF-1 target from a microarray study in which the transcriptional profiles of wild type and DIF signalling mutants were compared (our unpublished results; see Materials and methods). HfnA (DDB G0283983) contains a C-terminal HECT domain and an N-terminal filamin domain. Searches indicate that proteins containing both filamin and HECT domains are present in most organisms and adopt the same modular architecture (Fig. 1A). Alignments of the filamin domains of these proteins indicate that they have clear homology to those found in filamin proteins from humans and Dictyostelium (Fig. 1B). Furthermore, it is also apparent that these proteins share more similarities with each other than with true filamin proteins (Fig. $1 \mathrm{~B}, \mathrm{C})$, indicating that this is an evolutionarily conserved group of related proteins.

\section{$h f n A$ is induced by DIF and expressed in psto cells} In order to confirm that $h f n A$ exhibits DIF-dependent developmental expression, we generated an $h f n A$ reporter construct in which upstream $h f n A$ promoter sequences were used to drive the expression of lacZ. First, $h f n A$ expression was induced by DIF-1 in a monolayer culture of Dictyostelium cells (Fig. 2A). Secondly, we used upstream $h f n A$ promoter sequences to drive $l a c Z$ expression to test whether $h f n A$ exhibits DIF-1-dependent cell type-specific developmental regulation. Previous studies have shown that DIF1 acts on pstO cells, which are mainly localised to the collar region of the slug (Thompson and Kay, 2000). Accordingly, $h f n A$ expression was highly enriched in the pstO cell regions at the slug stage of wild-type development (Fig. 2B). Although expression levels are much reduced in culminants, any cells that did stain were found in the largely pstO cell-derived upper cup (Fig. 2B).

\section{Developing $h f n A^{-}$cells exhibit delayed culmination and increased slugging}

In order to determine the role of $\mathrm{HfnA}$ during development, we generated two independent $h f n A^{-}$mutants by homologous recombination. One mutant line was generated with a $3.5 \mathrm{~kb}$ 
A

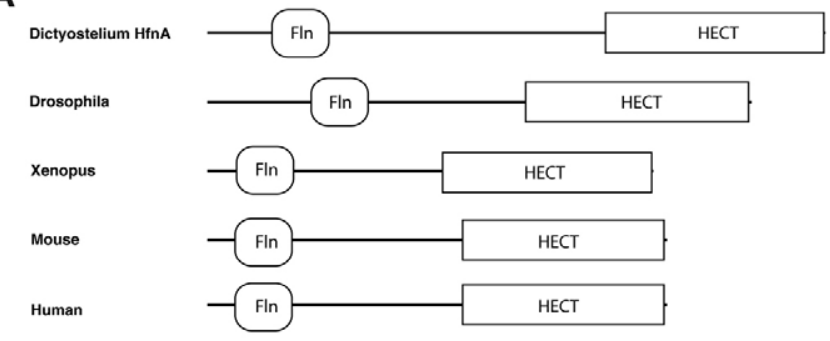

B Dictyostelium HfnA Drosophila
Xenopus Xenopus
Mouse Human $\underset{\text { Dictyostellum FIn }}{\text { Human FinA }}$

Dictyostelium HfnA Drosophila Xenopus Mouse Human FinA

Dictyostelium HinA Drosophila Mouse Human Human
Dictyostelium FIn
Human FinA
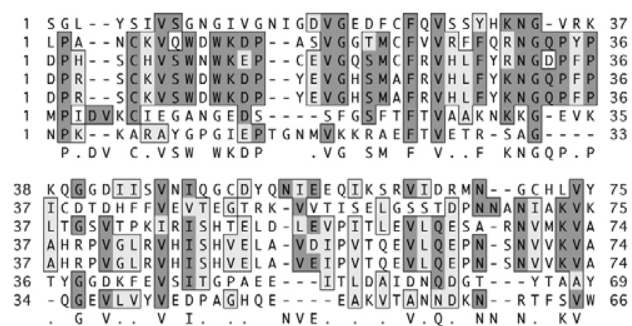

C

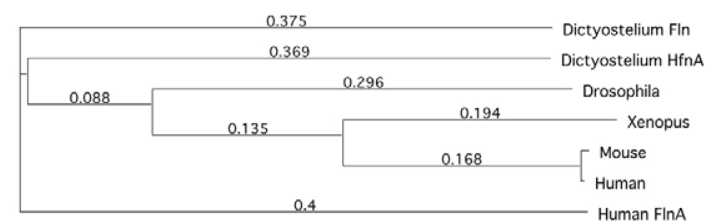

0.05

Fig. 1. An evolutionarily conserved group of HECT ligases containing an $\mathrm{N}$-terminal filamin domain. (A) A novel group of ubiquitin ligases that contain a C-terminal HECT domain and N-terminal filamin domains. Homologues from Dictyostelium, Drosophila (AAM29285), Xenopus (AAH94107), mouse (BAC36566) and human (AAF61276) are shown. (B) Alignment of the $\mathrm{N}$-terminal filamin domains from HECT ligase homologues from Dictyostelium, Drosophila, Xenopus, mouse and human, as well as Dictyostelium filamin (Fln domain 6) and human filamin A (FLNA domain 1). (C) Phylogenetic tree showing relatedness of filamin domain-containing HECT ligases from Dictyostelium, Drosophila, Xenopus, mouse and human, and their relationship to the true filamins Dictyostelium filamin (Fln domain 6) and human filamin A (FLNA domain 1). The bar represents 0.05 amino acid substitutions per site.

insertion at the beginning of the HECT domain and another with a large deletion within the $h f n A$ coding sequence that removes both the HECT and filamin domains (Fig. 3A). The mutants exhibit indistinguishable phenotypes and are thus likely to be null alleles. When developed on non-nutrient agar plates, $h f n A^{-}$ slugs appeared smaller and misshapen compared with wild type (Fig. 3B). The $h f n A^{-}$cells also showed a so-called 'slugger' phenotype (Sussman et al., 1978), in which culmination is delayed such that the slugs were still migrating after 24 hours of development on non-nutrient agar plates (Fig. 3B). When grown on Klebsiella lawns, the $h f n A^{-}$mutants also exhibited another manifestation of the slugger phenotype in that a large number of slug trails could be seen extending out from the edge of a growing plaque (Fig. 3C)
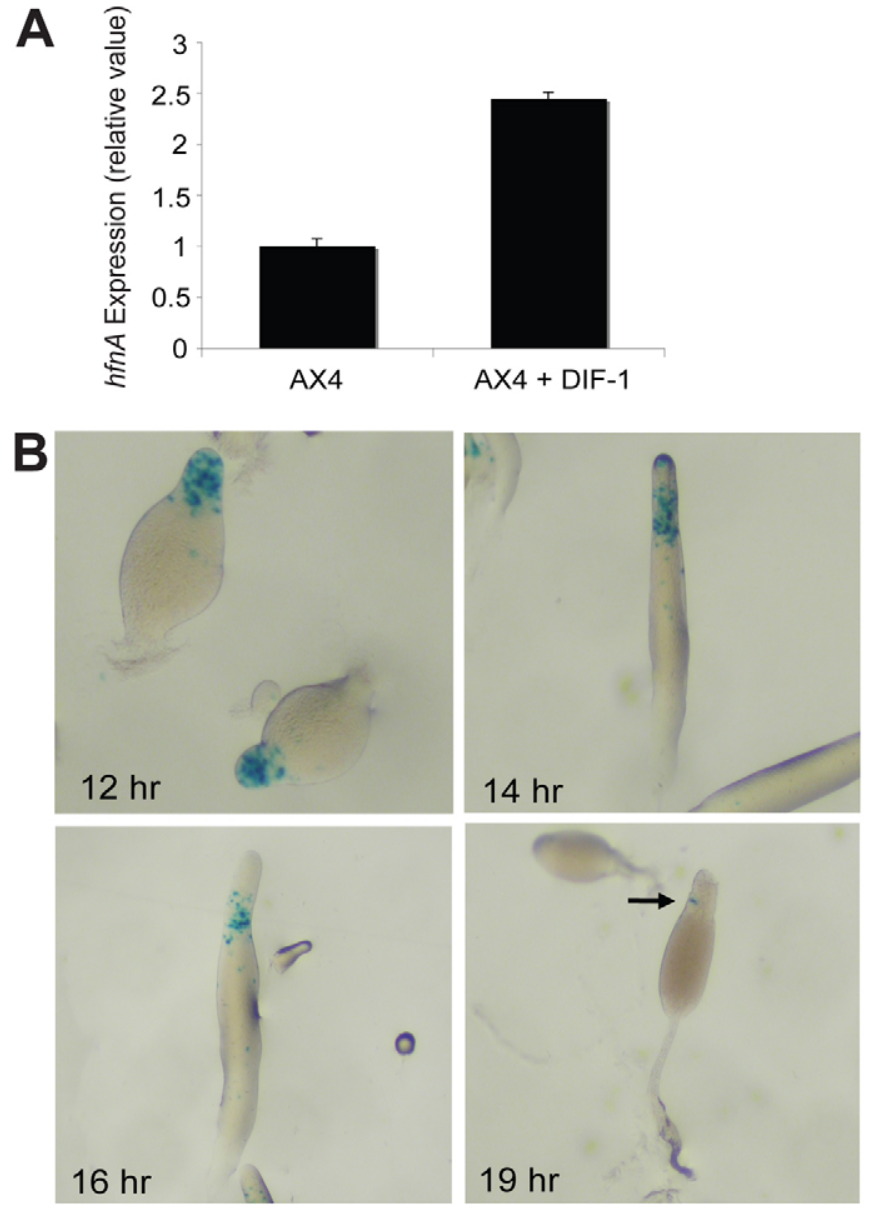

Fig. 2. $\boldsymbol{h f n} \boldsymbol{A}$ is a DIF-1 target. (A) DIF-1 induction of lacZ driven by the hfnA promoter in a Dictyostelium AX4 cell culture monolayer assay. Relative levels of $\beta$-galactosidase expression are shown with and without DIF-1 addition. Data are mean + s.d. (B) Localisation of $h f n A$ expression in the pstO region. Developing structures were fixed and stained for $\beta$-galactosidase expression (blue) driven by the $h f n A$ promoter at the indicated times post-transformation. The arrow indicates a single blue-stained cell in the (pstO) upper cup region.

\section{HfnA is required for normal psto cell sorting}

In order to investigate the cause of the $h f n A^{-}$mutant developmental phenotypes, we examined the expression of cell type-specific markers. As $h f n A$ expression is DIF-1 induced and $h f n A$ is expressed in pstO cells, this suggested a role in pstO cell differentiation or patterning. We found that ecmAO-lacZ (a marker of both pstA and pstO cells - the entire length of the prestalk region) strongly stained the slug tip, but was expressed more weakly behind this, in the pstO region (although scattered cells were also visible within the prespore zone), when $h f n A^{-}$ slugs were compared with wild type (Fig. 4). This staining pattern suggested a specific defect in pstO cell differentiation. Consistent with this, no defect in ecmA-lacZ expression (which normally stains the very tip of the slug - the pstA cells) could be detected (Fig. 4). Most importantly, ecmO-lacZ expression (a specific pstO cell marker that is normally expressed in the collar region behind the pstA cells) was clearly aberrant (Fig. 4). In the $h f n A^{-}$mutant, pstO cells were still visible but strikingly mislocalised towards 
A

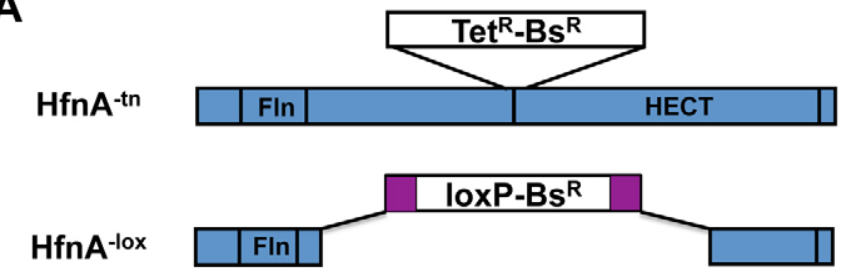

B
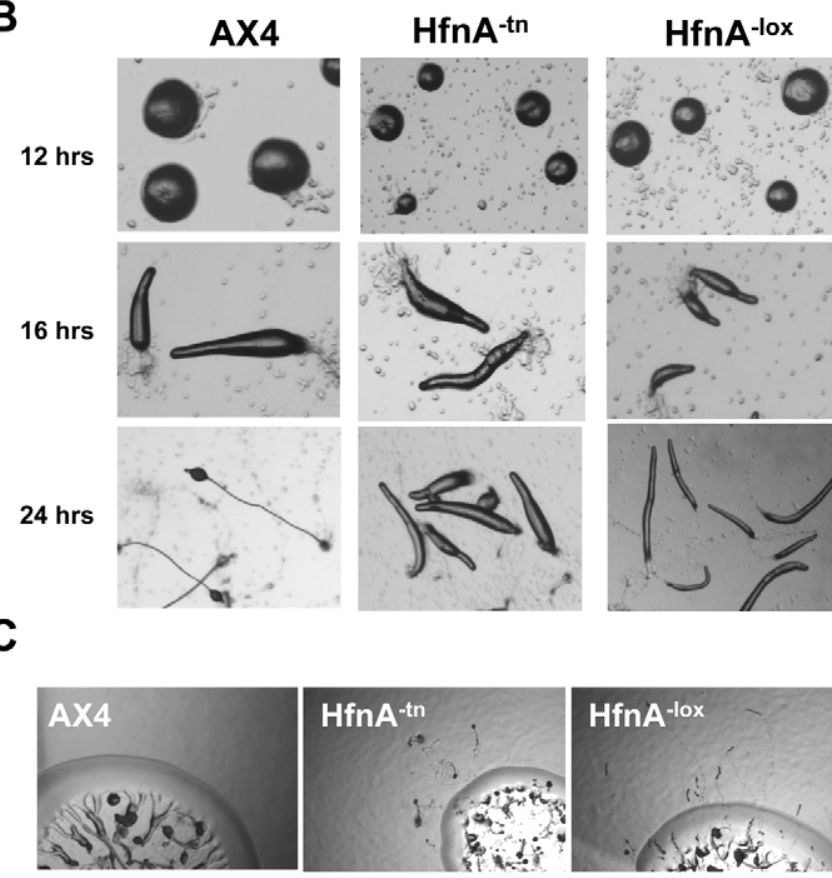

Fig. 3. $h f n A^{-}$cells exhibit delayed culmination and increased plaque 'slugging'. (A) Dictyostelium HfnA contains an N-terminal filamin repeat (FIn) and a C-terminal HECT domain (HECT). hfnA gene disruptions were made by inserting a transposon containing the blasticidin selectable marker into the $\mathrm{N}$-terminus of the HECT domain $\left(h f n A^{-t n}\right.$ ) or by replacing 717 amino acids of HfnA with a blasticidin selectable marker flanked by loxP sites (removed by transient expression of Cre recombinase) ( $h f n A^{- \text {lox }}$. (B) $h f n A^{-}$mutants exhibit delayed culmination and abnormal slug morphology. Wild-type (AX4) and $h f n A^{-}$ cells were developed on non-nutrient agar plates in the dark for 12, 16 and 24 hours. (C) When grown as plaques, the $h f n A^{-}$mutants exhibit increased slugging from the edge of the colony. Cells were spotted onto Klebsiella lawns and observed after 5 days growth in the light in a humid chamber.

the rear of the slugs. This defect is therefore distinct from that described for other DIF signalling mutants, in which pstO cells fail to differentiate.

\section{HfnA is required for psto cell targeting and motility}

As pstO cells fail to occupy the collar region at the early slug stage of development in the $h f n A^{-}$mutant, these findings suggest that $\mathrm{HfnA}$ is important for targeting or keeping the pstO cells in the correct place. To address this, we carefully followed the behaviour of pstO cells over a developmental time series. At the tight mound stage, the earliest stage at which ecmO-lacZ expression can be detected, staining was scattered in both wild type and mutant (Fig. 5). However, as soon as a tip could be

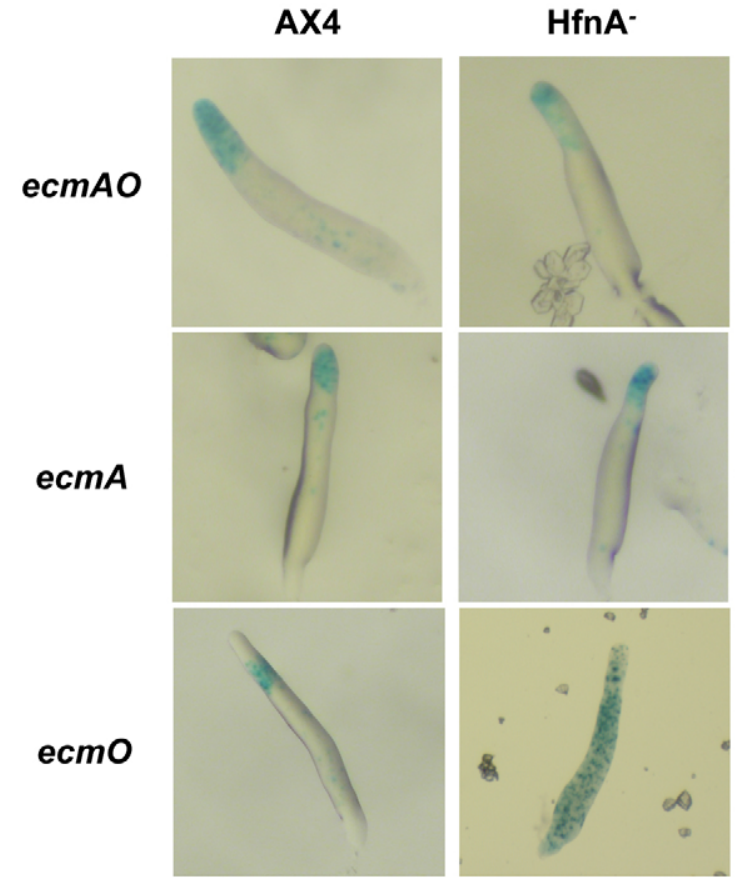

Fig. 4. HfnA is required for correct pstO cell localisation.

Dictyostelium slugs expressing ecmAO-lacZ, ecmO-lacZ and ecmA-lacZ were stained with $X$-gal to detect $\beta$-galactosidase expression (blue). Wild type (AX4) and $h f n A$-null mutants are shown. Mislocalisation of pstO cells is seen in the $h f n A^{-}$slugs.

detected, pstO cells were seen to accumulate below the tip in the wild type but not the $h f n A^{-}$mutant. This phenotype was most apparent at the early finger and slug stages. However, when slugs were examined over a timecourse, pstO cells were found in a pattern indistinguishable from that seen in wild type at the late slug stage (Fig. 5A). These findings therefore suggest that HfnA is needed for the correct early targeting or motility of pstO cells to the collar region of the developing finger and early slug, as pstO cells are mislocalised in the $h f n A^{-}$mutant (Fig. 5B).

It is thought that prestalk cell sorting is driven by differential adhesion and/or chemotaxis towards a localised source of cAMP produced by the developing tip cells. If so, the delay in pstO cell sorting could be due to their failure to sense and orient to the chemoattractant stimulus, or to a simple failure to move within the three-dimensional environment in the mound (or some combination of the two). To distinguish between these possibilities, we first directly measured the speed and directionality of prestalk and prespore cell movement, using real-time imaging, during the transition from mound to finger stages. In order to follow pstO cell movement, co-transformant lines were generated that express ecmAO-RFP and ecmO-GFP. Cells expressing both markers were defined as pstO cells. For prespore cells, psA-RFP transformants were used. PstO and prespore cells were tracked to determine their speed and directionality of movement. From this, we found that mutant pstO cells move at a significantly slower speed, which was almost half that of wild-type controls (Fig. 6A). This is consistent with the finding that complete pstO sorting takes $\sim 6-8$ hours in the mutant compared with 3-4 hours in the wild type (Fig. 5A). More importantly, this does not reflect a general motility defect in the mutant, as the speed of prespore movement was indistinguishable 
A
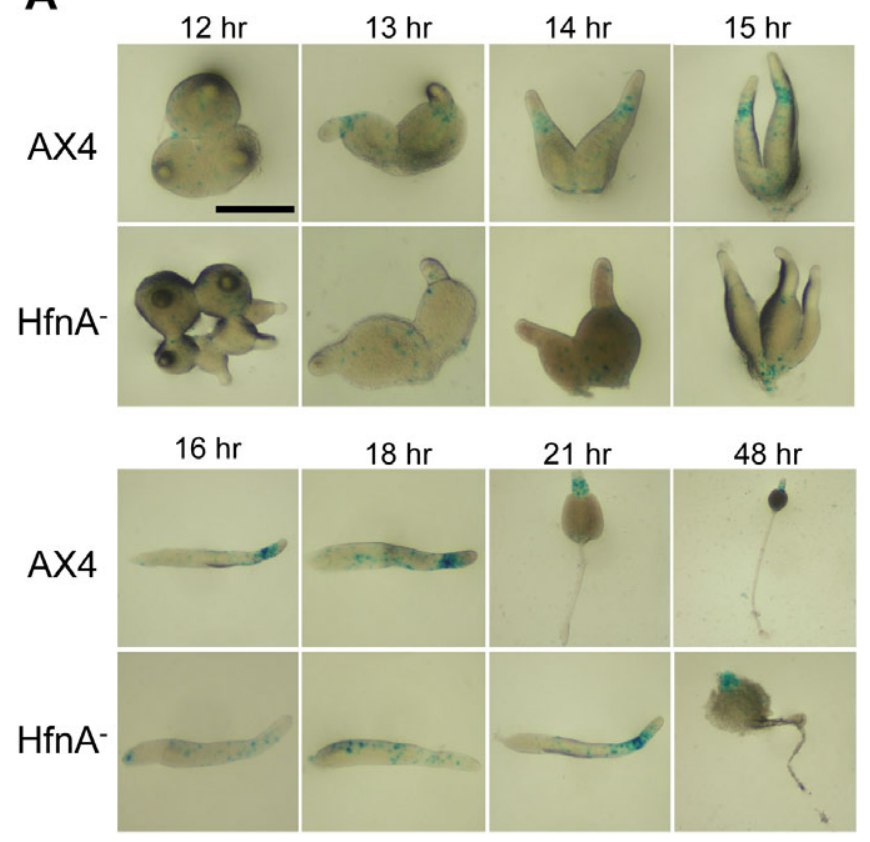

B

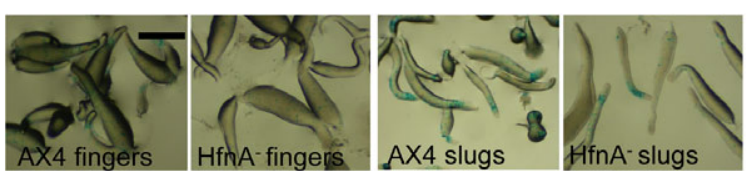

Fig. 5. PstO cell sorting defects in the $h f n A^{-}$mutant are only seen at early stages of development. Wild-type and mutant Dictyostelium expressing ecmO-lac $Z$ were stained at the tight mound, tip mound, early finger, early slug and late slug stages. (A) $h f n A^{-}$pstO cells are only mislocalised at early stages of development (12-18 hours) and eventually reach the collar much later (21 hours), as compared with wild-type AX4 cells. (B) A selection of wild-type and $h f n A^{-}$specimens expressing ecmO-lac $Z$ at finger and slug stages. Scale bars: $0.5 \mathrm{~mm}$.

between the wild type and mutant (Fig. 6B). Finally, and as expected, the speed of pstO cell movement was significantly higher than that of prespore cells, although the difference was not as marked in the $h f n A^{-}$mutant as in the wild type (Fig. 6A,B). Importantly, however, when the directionality of movement of each cell type was compared, no differences between wild-type and mutant cells were detected (Fig. 6A,B).

Since the speed, but not directionality, of movement of mutant pstO cells was affected, this suggested a defect in cell motility but not chemoattractant sensing. In order to confirm this we compared the ability of dissociated wild-type and $h f n A^{-}$pstO cells to move towards a micropipette containing cAMP. Under these conditions, we detected no defect in the speed or directionality of movement of the mutant pstO cells, supporting the idea that the ability of $h f n A^{-}$cells to sense a chemoattractant is unaffected (Fig. 6C). Furthermore, these findings suggest that the defect is specific to movement in a three-dimensional environment. Since both differential adhesion and chemotaxis have been implicated in prestalk cell sorting during normal development, we tested whether adhesion might be affected. Wild-type and $h f n A^{-}$cells expressing the prestalk marker ecmAO-RFP were dissociated at the finger stage of development
A
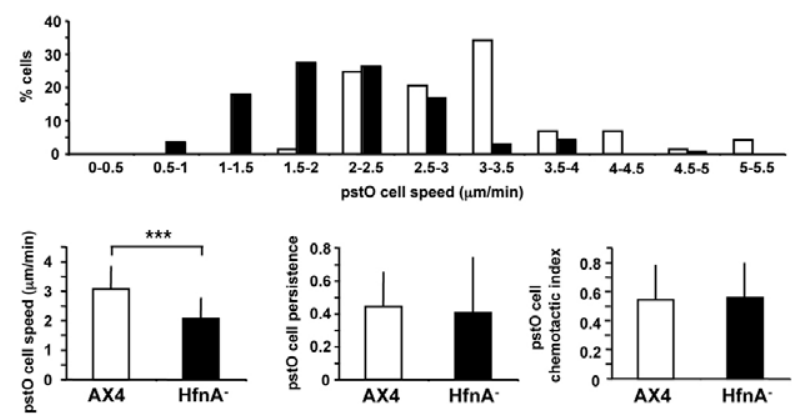

B
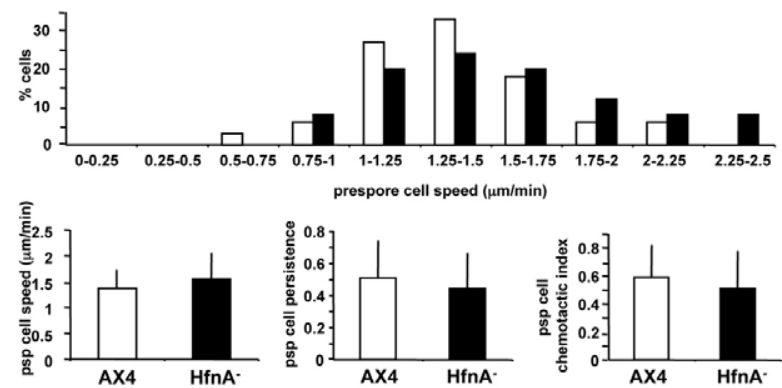

C
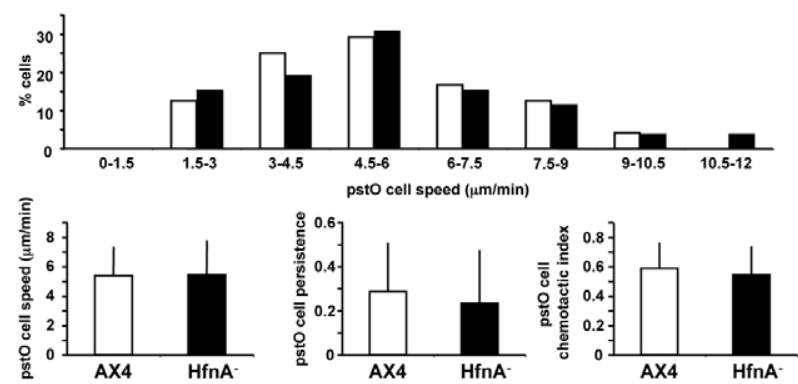

Fig. 6. HfnA regulates the speed, but not directionality, of pstO cell migration. (A-C) PstO (A) and prespore (B) cell movement during normal Dictyostelium development, and movement of dissociated pstO cells to a micropipette containing CAMP (C). To follow pstO cell movement, co-transformant lines were generated that express ecmAORFP and ecmO-GFP. Cells expressing both markers were defined as pstO cells. For prespore cells, psA-RFP transformants were used. The distribution of cell speeds is shown for the wild type and $h f n A^{-}$mutant. Comparisons of cell speed, chemotaxis index and persistence are also shown. For pstO mound movement, data from six AX4 movies (75 cells) and ten $h f n A^{-}$movies (169 cells) were pooled. For prespore mound movement, data from two AX4 movies (35 cells) and two $h f n A^{-}$movies ( 25 cells) were pooled. For dissociated pstO cell speed, data from eight AX4 movies ( 25 cells) and six $h f n A^{-}$movies ( 27 cells) were pooled. Data are mean + s.d. ANOVA analysis revealed significant differences between pstO and prespore speeds, and between $\mathrm{AX} 4$ and $h f n A^{-}$pstO cell speeds $(* * *, P<0.001)$. No other statistically significant differences between pairings were observed.

and allowed to reaggregate in suspension. The extent of prestalk cell aggregation was indistinguishable between wild-type and mutant cells (Fig. 7). This indicates that neither the adhesive properties nor the chemotactic responses of mutant prestalk cells are affected. Taken together, these findings suggest that the defect in pstO cell sorting in the $h f n A^{-}$mutant is most likely due to a failure to drive movement in the three-dimensional environment of multicellular development. 


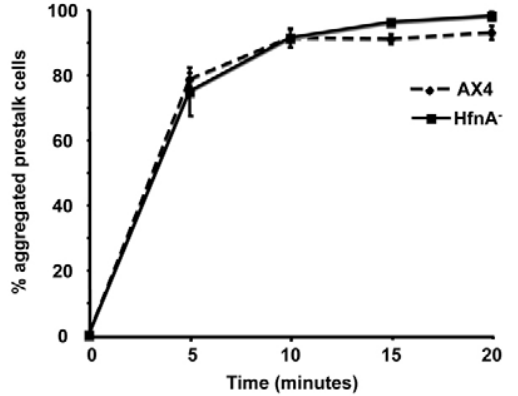

Fig. 7. Prestalk cell adhesion is unaffected in the $h f n A^{-}$mutant. Cell-cell adhesion of finger stage cells was measured by dissociating and counting the number of cells that had reaggregated at different time points. AX4 and $h f n A^{-}$ecmAO-RFP-expressing cell reaggregation are shown. Error bars indicate s.e.

\section{Changes in filamin complex activity affect psto cell patterning}

HECT domain-containing ubiquitin ligases often contain a proposed N-terminal substrate-targeting domain (Scheffner and Staub, 2007). HfnA contains an N-terminal filamin domain, which is rarely found in Dictyostelium proteins. We therefore reasoned that filamin, or filamin complex members, could be targets of HfnA during development. Indeed, a role for filamin in developmental patterning has previously been suggested because Fln-null cells fail to sort to the tip of slugs when developed in chimera with wild-type cells. A simple hypothesis, therefore, is that aspects of the $h f n A^{-}$phenotype are caused by increased filamin, or filamin complex, activity. To test whether filamin levels were altered, we analysed the amount of filamin present in $h f n A^{-}$versus wild-type cells at different developmental stages (Fig. 8). However, we found no significant differences in filamin levels between the wild type and $h f n A^{-}$mutant.

Another possibility is that HfnA targets a filamin-interacting protein, thereby affecting the activity of filamin complexes. To address this, we tested for genetic interactions by examining the effects of increasing or decreasing filamin complex activity in wild-type or $h f n A^{-}$genetic backgrounds. First, we found that disruption of the filamin gene $\operatorname{abpC}\left(\mathrm{Fln}^{-}\right.$; loss of filamin complex activity) in the $h f n A^{-}$background $\left(h f n A^{-} / F l n^{-}\right)$effectively rescues the slug morphology and delayed culmination defects of the $h f n A^{-}$ mutant (Fig. 9A). Second, we found that filamin disruption results in the 'opposite' pstO cell phenotype to that caused by $h f n A$ disruption: PstO cells were more strongly localised, resulting in a tight collar with few, if any, scattered cells in the rear of slugs (Fig. 9B). Furthermore, filamin disruption in an $h f n A^{-}$background resulted in an intermediate 'rescued' phenotype, thus demonstrating a genetic interaction between $h f n A$ and $F l n$ (Fig. 9B). Finally, overexpression of filamin or the C-terminal portion of the known filamin binding partner FIP (Knuth et al., 2004) in the wild type led to pstO mislocalisation (Fig. 9C). The pstO sorting defect seen in the $h f n A^{-}$mutant is thus phenocopied when filamin complex activity is artificially elevated. It is also interesting that the effects of increasing filamin complex activity through FIP overexpression do not depend on the presence of filamin, as they are observed in a Fln-null background (Fig. 9C). This supports the idea that the effects are due to misregulation of filamin complex activity rather than of filamin activity itself. Most importantly, as filamin is an actin-binding adapter protein that is
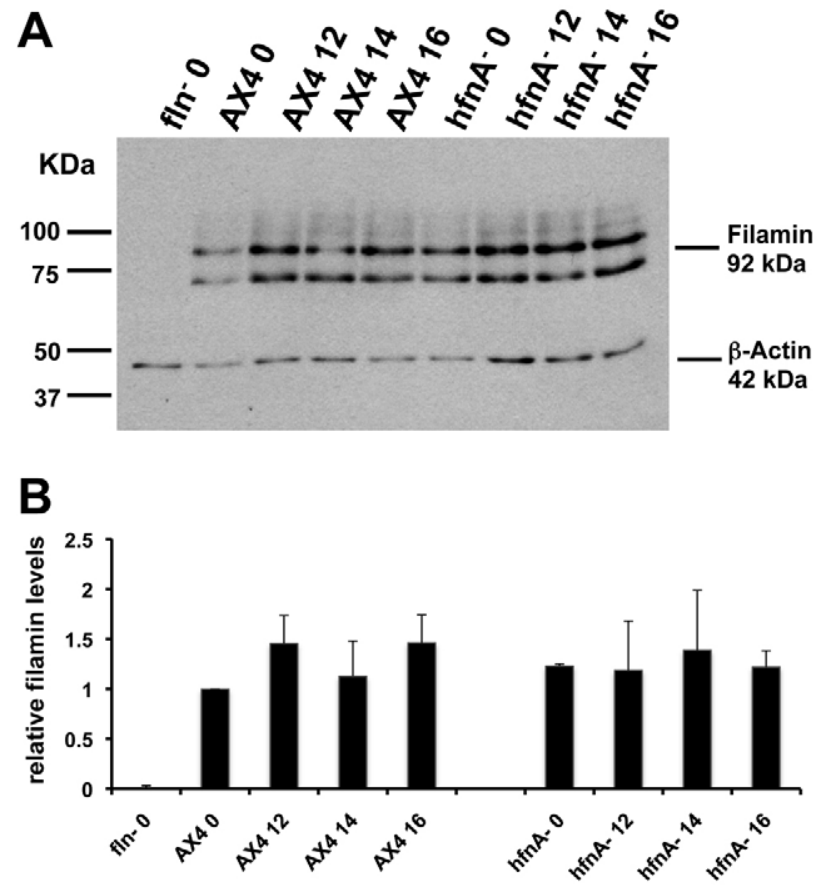

Fig. 8. The $h f n A^{-}$phenotype is dependent on filamin activity not filamin levels. (A) Filamin levels are unchanged in the $h f n A^{-}$mutant. Western blot showing filamin levels at different stages of development in AX4 and $h f n A^{-}$mutant. Numbers refer to developmental time point (hours). $\beta$-actin is included as a loading control. The Fln-null mutant extract illustrates the specificity of the antibody. Protein extracts were normalised for total protein and $10 \mu \mathrm{g}$ was loaded in each case. (B) Quantification of filamin levels from two independent experiments as in A. Filamin levels were normalised to the $\beta$-actin loading control. Data are mean + s.d.

known to be involved in cell movement and adhesion (Popowicz et al., 2006), altered filamin complex activity in the $h f n A^{-}$mutant provides an explanation for the observed mislocalisation of $\mathrm{pstO}$ cells.

\section{Photosensory responses are enhanced by the absence of HfnA}

Filamin is essential for slug phototaxis and filamin-deficient strains exhibit severe phototaxis defects (Fisher et al., 1997; Khaire et al., 2007; Knuth et al., 2004). It is thought that filamin is present in a 'phototaxis complex' that is involved in sensing light and in signal transduction at the tip of the slug (BandalaSanchez et al., 2006). We tested whether HfnA is also required to regulate normal responses to light. Interestingly, the slugging phenotype of the $h f n A^{-}$mutant requires a lateral light source, as incubating the mutants in the dark resulted in no slugging at all from the edge of the plaque (Fig. 10). As wild-type slugs do not usually migrate out of the plaque, even in the light, $h f n A^{-}$slugs appear more sensitive to light. This phenotype is consistent with hyperactivity of the filamin complex, as filamin has been shown to be required for slug phototaxis. Consistent with this idea, we found that the slugging phenotype was rescued in the $h f n A^{-}$ mutant by inactivation of filamin (Fig. 9). To confirm this, we tested the ability of $h f n A^{-}$slugs to orient accurately during phototaxis. We found that phototaxis by $h f n A^{-}$slugs was enhanced compared with the parental wild-type controls (Fig. 
A

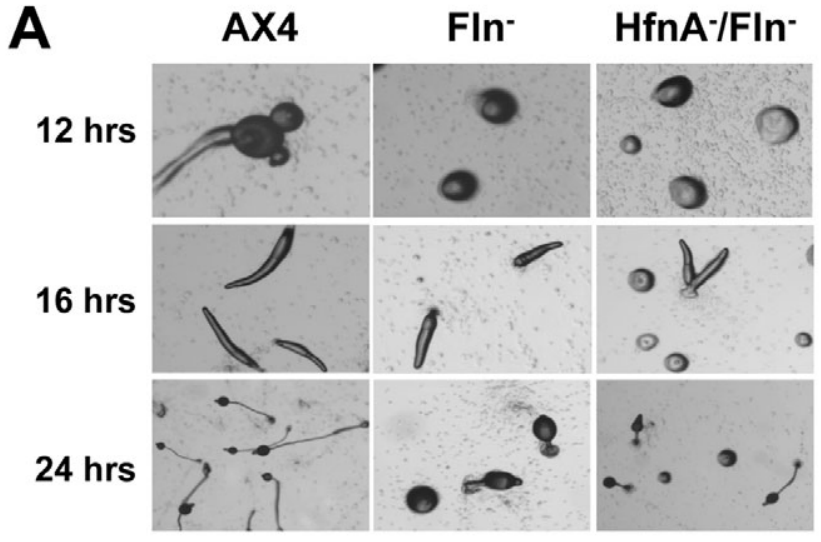

B

AX4

ecmAO

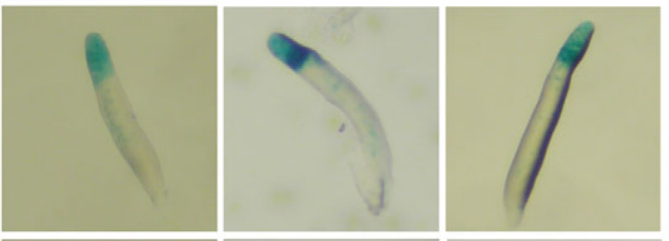

ecmA

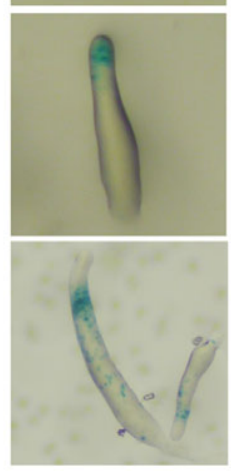

C

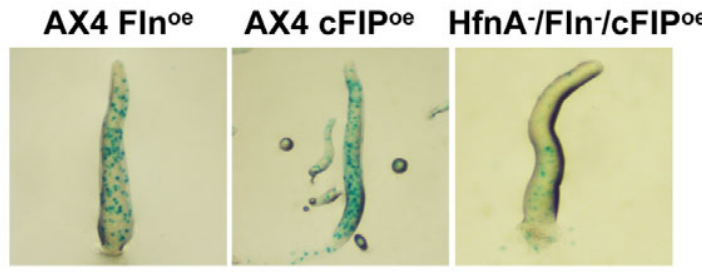

Fig. 9. Genetic interaction between $h f n A$ and Fln reveals that changes in filamin complex activity affect pstO cell localisation. (A) Disruption of filamin rescues the slugging and culmination defects of the $h$ fnA- mutant. (B) pstO cell localisation in the absence of filamin activity. Disruption of filamin results in tighter localisation of pstO cells to the collar. A few scattered pstO cells can be seen in the rear of slugs. When filamin levels are decreased in the $h f n A^{-}$mutant $\left(h f n A^{-} / F / n^{-}\right.$ double mutant), an intermediate phenotype is observed. This is most evident by the large increase in ecmO-lacZ-expressing cells in the rear in the double mutant. (C) Overexpression of filamin complex members results in mislocalisation of pstO cells in Ax4 wild-type, $h f n A^{-}$and hfn $A^{-} / F / n^{-}$mutant slugs.

11). In quantitative experiments, the accuracy of phototaxis by $h f n A^{-}$slugs was $\sim 1.5$-fold greater than that of the wild type (Fig. $12 \mathrm{~A}$ and Table 1). Since phototaxis accuracy depends quantitatively on filamin complex activity (Annesley et al., 2007), this suggests signalling hyperactivity by the filamin complex when HfnA is absent. As expected, $h f n A^{-} / F l^{-}$slugs,

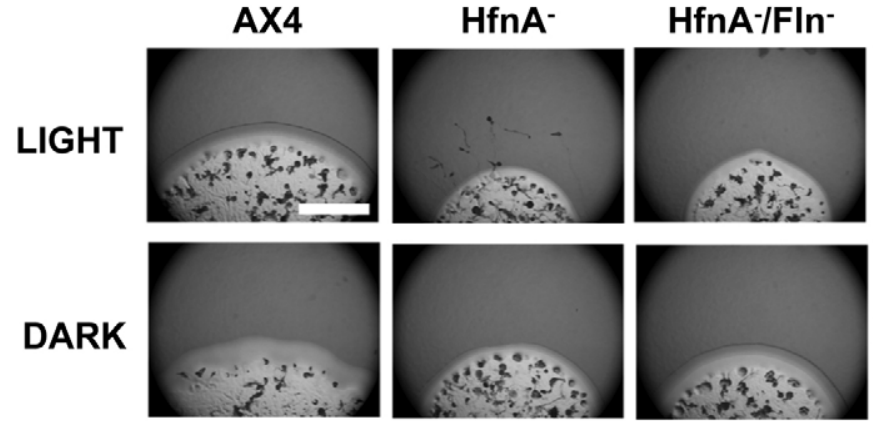

Fig. 10. HfnA suppresses slug migration in response to a lateral light source. Light is required for the $h f n A^{-}$slugging phenotype. Cells were spotted onto Klebsiella lawns and observed after 5 days in light and dark conditions. Incubation in light caused numerous slug trails to extend from the feeding edge of $h f n A^{-}$plaques. Incubation in the dark suppressed this phenotype, indicating that this slugging requires light. The slugging phenotype is suppressed by removal of filamin function $\left(h f n A^{-} / F / n^{-}\right)$. Scale bar: $0.5 \mathrm{~cm}$.

like Fln-null mutants, exhibited severe phototaxis defects. Finally, although cFIP has previously been reported to show dominant-negative effects on filamin complex activity and thus impair phototaxis, we found that overexpression of cFIP in the genetic background used in this study improves phototaxis efficiency (Figs 11 and 12, Table 1). Together, these findings provide further support for the idea that HfnA inhibits the signalling activity of the filamin complex.

\section{DISCUSSION \\ HfnA is required for correct patterning via regulation of filamin complexes}

Our findings provide a crucial mechanistic advance in our understanding of pattern formation by salt-and-pepper differentiation and sorting out in Dictyostelium. It has been proposed that heterogeneities in sensitivity to the DIF-1 signal within cell populations generate cell type-specific gene expression, which in turn drives cell sorting (Kay and Thompson, 2009). Our study identifies the first such gene, $h f n A$. HfnA is the first member to be characterised of a novel group of evolutionarily conserved proteins, the HECT ligases, which contain an N-terminal filamin domain. In Dictyostelium, HfnA is DIF induced, expressed in pstO cells and essential for normal development. We have demonstrated that HfnA is important for the timing of culmination, the regulation of slug movement and localisation of pstO cells. $\mathrm{HfnA}$ appears to regulate the activity of filamin complexes, which are in turn needed for phototactic slug migration and correct cell type motility and patterning during development. These studies provide the first step in defining the molecular relationship between the input DIF-1 signal and output cell type-specific behaviours that lead to sorting out.

Pattern formation is a complex and coordinated process. $\mathrm{HfnA}$, through its downregulation of specific targets, is essential for normal development. We have demonstrated that filamin is a possible target for $\mathrm{HfnA}$, as filamin disruption rescues, and filamin hyperactivation phenocopies, the pstO sorting and slug phototaxis phenotypes of the $h f n A^{-}$mutant. Despite this genetic link, we found that filamin levels are unchanged in the $h f n A^{-}$ mutant. One possibility is that any change in filamin level might be extremely small as it may be restricted to the pstO cell 


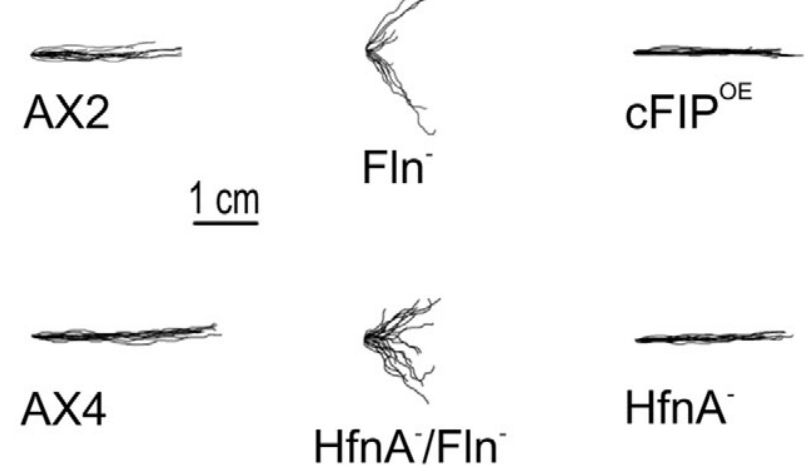

Fig. 11. HfnA restricts the accuracy of slug phototaxis responses. Digitised slug trails of two wild-type strains, AX4 and AX2, hfnA ${ }^{-}$, Fln-, C-terminal fragment of FIP (CFIP) overexpression strain, and $h f n A / F / n^{-}$. AX4 is the parental strain for the $h f n A^{-}$and $h f n A^{-} / F / n^{-}$mutants, whereas AX2 is the parental strain for the Fln null. Fln knockout and cFIP overexpression produced the same phenotypes in the alternate wild-type genetic backgrounds (not shown). Slug trails were plotted from a common origin so that the source of light is to the right in the images. The slugs of both wild-type strains migrate directly towards the light source, whereas the Fln-null and the $h f n A^{-} / F / n^{-}$slugs show disoriented, bimodal phototaxis with two preferred directions either side of the light source. The $h f n A^{-}$strain shows slightly improved phototaxis, such that the slugs move more directly towards the light source than the wild-type strains ( $X \times 2$ and AX4).

population of the slug. However, we found that the expression of a C-terminal fragment of FIP phenocopies a lack of HfnA. FIP levels are, however, unchanged in the $h f n A^{-}$mutant (data not shown). Therefore, our data support an alternative explanation in which neither filamin nor FIP is the direct target of HfnA. Instead, other filamin interactors might be targeted, leading to altered filamin complex activity. The identification of these putative targets is likely to further reveal the roles of HfnA in DIF-1-dependent patterning events.

Table 1. Phototaxis is enhanced by loss of HfnA or by overexpression of cFIP

\begin{tabular}{|c|c|}
\hline Strain & Phototaxis \\
\hline $\mathrm{AX} 2$ & $109 \pm 13,119 \pm 25$ \\
\hline$\times 4$ & 22 \\
\hline $\mathrm{AX} 4 / h f n A^{-}$ & $166 \pm 28 *$ \\
\hline $\mathrm{AX} 4 / h f n A^{-} / F / n^{-}$ & $2.24 \pm 0.36 * *$ \\
\hline $\mathrm{A} \times 2 / F / n^{-}$ & $25 * *$ \\
\hline $\mathrm{AX} 4\left(\mathrm{CFIP} \mathrm{OE}^{-}\right)$ & $183 \pm 27$ * \\
\hline $\mathrm{AX} 4 / h f n A^{-}(\mathrm{cFIPOE})$ & $146 \pm 21 *$ \\
\hline $\mathrm{AX} 4 / h f n A^{-} / F / n^{-}\left(\mathrm{cFIP}{ }^{\mathrm{OE}}\right)$ & $1.79 \pm 0.28 * *$ \\
\hline \multicolumn{2}{|c|}{ 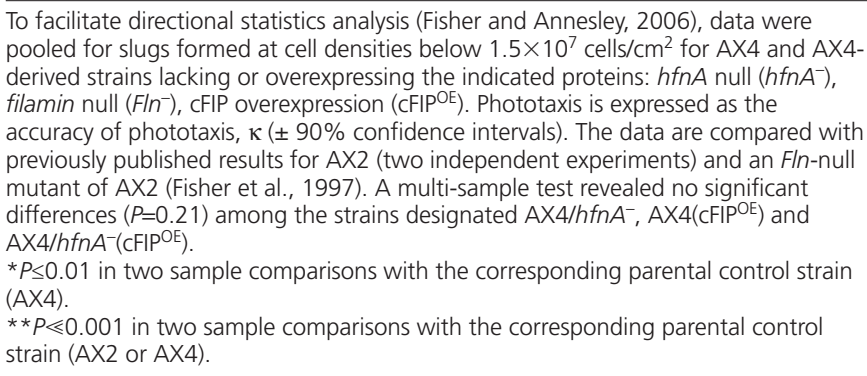 } \\
\hline
\end{tabular}
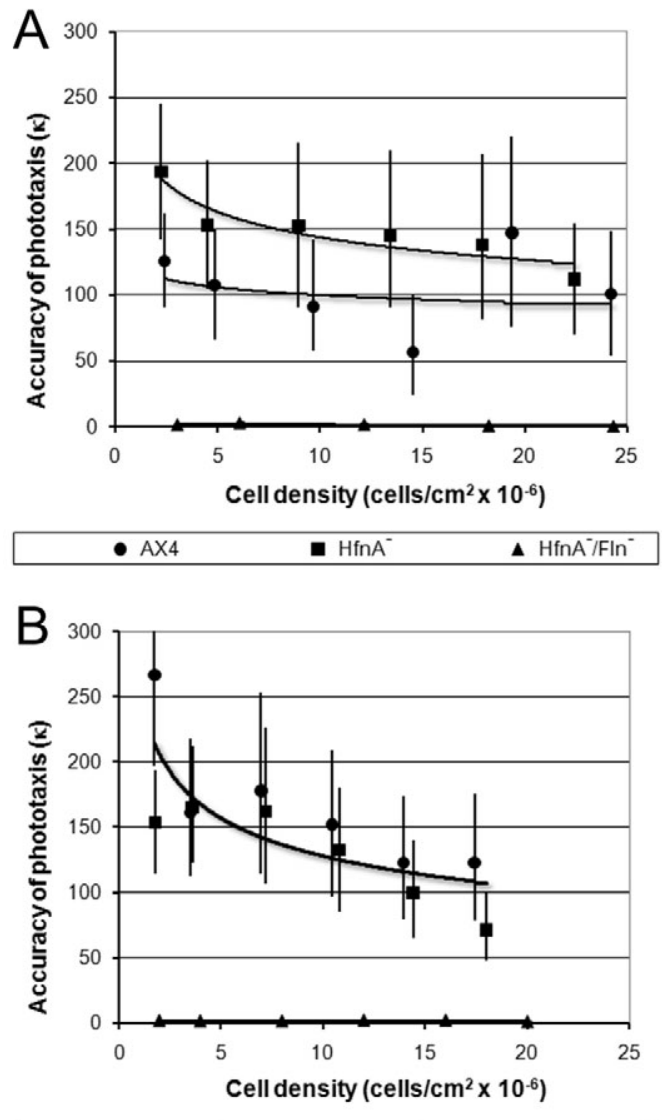

- $\mathrm{AX} 4\left(\mathrm{CFIP}^{\circ E}\right)$ - $\mathrm{HfnA}^{-}\left(\mathrm{CFIP}^{\sigma E}\right) \quad \boldsymbol{\Delta} \mathrm{HfnA}^{-} \mathrm{Fln}^{-}\left(\mathrm{CFIP}^{\circ E}\right)$

Fig. 12. Both cFIP overexpression and $h f n A$ knockout result in improved phototaxis. (A,B) Quantitative phototaxis was performed at defined cell densities on wild-type AX4, $h f n A^{-}$and $h f n A^{-} / F / n^{-}$mutants (A) and on the same strains overexpressing CFIP (B). The trails were digitised and analysed using directional statistics to determine their accuracies of phototaxis ( $\kappa$ ) at the defined cell densities. Vertical bars are $90 \%$ confidence limits. Except at cell densities greater than $1.5 \times 10^{7} \mathrm{cells} / \mathrm{cm}^{2}$, the $h f n A^{-}$and cFIP-overexpressing strains both displayed higher accuracies of phototaxis than the parental wild-type AX4 (differences significant at $P<0.01$ in log-linear regression analysis), whereas the $h f n A^{-} / F / n^{-}$double mutant displayed poor accuracies of phototaxis whether or not CFIP was expressed. The $h f n A^{-} / F / n^{-}$strain showed accuracies of phototaxis that were $\sim 1 \%$ of AX4 wild-type levels. The $h f n A^{-}$and cFIP-overexpressing strains showed accuracies of phototaxis that were $\sim 150 \%$ of AX4 wild-type levels. Log-linear regression analysis detected no significant difference $(P>0.1)$ between AX4 (cFIPOE) and $h f n A^{-}(\mathrm{CFIPOE})$, so the line of best fit in B was plotted using data from both strains.

\section{Filamin and the regulation of cell movement during multicellular development}

Cell type-specific sorting in Dictyostelium is thought to require both differential cell motility and adhesion. Cells must respond to the cAMP chemoattractant produced by the tip and navigate their way through the three-dimensional environment of other cells. Furthermore, the different prestalk subtypes must also behave differently in order for relatively complex cell type organisation to arise. A possible mechanism to explain this is that all cells have an equal ability to sense the chemoattractant but move at different speeds or with different strengths. For example, by modulating adhesion or intercellular contacts, it is possible to envisage a 
situation in which the most adhesive cells move more slowly because they are, in effect, tethered. Alternatively, each cell type could differ in the motile force generated in response to a directional signal. Our data support a role for the filamin complex in the regulation of cell type-specific speed of movement, but not in adhesion or direction sensing. Since the filamin complex is known to regulate actin cytoskeleton dynamics, we suggest that this might underlie the pstO sorting defects observed. Indeed, some support for this idea comes from the finding that other mutants with defects in cytoskeletal organisation exhibit cell type sorting defects, especially when developed in chimera with wild-type cells (Chisholm and Firtel, 2004).

\section{HECT ligases in Dictyostelium}

Ubiquitylation represents an evolutionarily conserved regulatory mechanism. RING finger ubiquitin ligases and ancillary proteins have been shown to be important for normal Dictyostelium development (Clark et al., 1997; Ennis et al., 2000; Nelson et al., 2000; Wang and Kuspa, 2002; Whitney et al., 2006) and even for slug phototactic migration (Whitney et al., 2006). Searches indicate that the Dictyostelium genome is likely to encode six HECT ligases, each containing a C-terminal HECT domain and most an identifiable $\mathrm{N}$-terminal domain that might be responsible for binding to its specific target. These $\mathrm{N}$-terminal domains include $\mathrm{PH}$ domains (DDB0187197), protein kinase and RCC1 domains (DDB0219986) and HEAT/Armadillo repeats (DDB0235186). Our studies provide the first characterisation of a Dictyostelium HECT ligase and suggest that in this case an $\mathrm{N}$-terminal filamin domain specifically targets filamin complexes. It will be of great interest to define the role of other HECT ligases in Dictyostelium, as these studies might also uncover evolutionarily conserved regulatory mechanisms.

\section{Filamin complexes and their regulation}

Filamins are adaptor proteins that have numerous binding partners and are able to bring together many signalling and cytoskeletal elements. The precise regulation of filamin activity is of crucial importance for normal filamin complex function. Consequently, filamin has multiple modes of regulation. Protein stability is central to the regulation of filamin complex activity. For example, filamin itself is subject to degradation by calpain (Onji et al., 1987). Filamin is also phosphorylated by a number of serine-threonine kinases, and its phosphorylation by protein kinase A inhibits its cleavage by calpain (Chen and Stracher, 1989). The importance of protein stability in its regulation is further illustrated by its mammalian binding partner FILIP (filamin A interacting protein), which also downregulates filamin through degradation (Nagano et al., 2002). Despite stability being a core theme in the regulation of filamin complex activity, interactions with ubiquitin ligases and the ubiquitylation system have not previously been demonstrated. For example, although ubiquitin ligases that contain both a RING finger and a filamin domain are found in some organisms (Schaefer et al., 2000; Wan et al., 2000), no role in filamin complex regulation has been described. Most importantly, we have found that a family of HECT ligase- and filamin repeat-containing proteins closely related to HfnA is widely conserved through evolution. We therefore propose that the degradation of filamin interactors via the proteasome might represent a novel and widespread mechanism for regulating filamin complex activity.

\section{Acknowledgements}

We thank Prof. Gad Shaulsky for help in conceiving the project and encouragement throughout, Neil Buttery for help with statistical analyses, and Dr Angelika Noegel for providing the filamin antibody and the cFIP construct. This work was supported by project grants from the Wellcome Trust and Medical Research Council, a Lister Institute of Preventive Medicine Research Prize to C.R.L.T. and funds to P.R.F. from the Thyne Reid Memorial Trusts. Deposited in PMC for release after 6 months.

\section{Competing interests statement}

The authors declare no competing financial interests.

\section{References}

Abe, T., Langenick, J. and Williams, J. G. (2003). Rapid generation of gene disruption constructs by in vitro transposition and identification of a Dictyostelium protein kinase that regulates its rate of growth and development. Nucleic Acids Res. 31, 107-111.

Annesley, S. J., Bandala-Sanchez, E., Ahmed, A. U. and Fisher, P. R. (2007). Filamin repeat segments required for photosensory signalling in Dictyostelium discoideum. BMC Cell Biol. 8, 48

Bandala-Sanchez, E., Annesley, S. J. and Fisher, P. R. (2006). A phototaxis signalling complex in Dictyostelium discoideum. Eur. J. Cell Biol. 85, 1099-1106.

Berry, F. B., O'Neill, M. A., Coca-Prados, M. and Walter, M. A. (2005). FOXC1 transcriptional regulatory activity is impaired by PBX1 in a filamin A-mediated manner. Mol. Cell. Biol. 25, 1415-1424.

Chazaud, C., Yamanaka, Y., Pawson, T. and Rossant, J. (2006). Early lineage segregation between epiblast and primitive endoderm in mouse blastocysts through the Grb2-MAPK pathway. Dev. Cell 10, 615-624.

Chen, M. and Stracher, A. (1989). In situ phosphorylation of platelet actinbinding protein by CAMP-dependent protein kinase stabilizes it against proteolysis by calpain. J. Biol. Chem. 264, 14282-14289.

Chisholm, J. C. and Houliston, E. (1987). Cytokeratin filament assembly in the preimplantation mouse embryo. Development 101, 565-582.

Chisholm, R. L. and Firtel, R. A. (2004). Insights into morphogenesis from a simple developmental system. Nat. Rev. Mol. Cell Biol. 5, 531-541.

Clark, A., Nomura, A., Mohanty, S. and Firtel, R. A. (1997). A ubiquitinconjugating enzyme is essential for developmental transitions in Dictyostelium. Mol. Biol. Cell 8, 1989-2002.

Clow, P. A., Chen, T., Chisholm, R. L. and McNally, J. G. (2000). Threedimensional in vivo analysis of Dictyostelium mounds reveals directional sorting of prestalk cells and defines a role for the myosin II regulatory light chain in prestalk cell sorting and tip protrusion. Development 127, 2715-2728.

Darcy, P. K., Wilczynska, Z. and Fisher, P. R. (1994). Genetic analysis of Dictyostelium slug phototaxis mutants. Genetics 137, 977-985.

Dietrich, J. E. and Hiiragi, T. (2007). Stochastic patterning in the mouse preimplantation embryo. Development 134, 4219-4231.

Dingermann, T., Reindl, N., Werner, H., Hildebrandt, M., Nellen, W., Harwood, A., Williams, J. and Nerke, K. (1989). Optimization and in situ detection of Escherichia coli beta-galactosidase gene expression in Dictyostelium discoideum. Gene 85, 353-362.

Early, A., Abe, T. and Williams, J. (1995). Evidence for positional differentiation of prestalk cells and for a morphogenetic gradient in Dictyostelium. Cell 83, 9199.

Egelhoff, T. T., Brown, S. S., Manstein, D. J. and Spudich, J. A. (1989). Hygromycin resistance as a selectable marker in Dictyostelium discoideum. Mol. Cell. Biol. 9, 1965-1968.

Ennis, H. L., Dao, D. N., Pukatzki, S. U. and Kessin, R. H. (2000). Dictyostelium amoebae lacking an F-box protein form spores rather than stalk in chimeras with wild type. Proc. Natl. Acad. Sci. USA 97, 3292-3297.

Fisher, P. R. and Annesley, S. J. (2006). Slug phototaxis, thermotaxis and spontaneous turning behaviour. In Dictyostelium discoideum Protocols. Methods in Molecular Biology, Vol. 346 (ed. L. Eichinger and F. Rivero), pp. 137-170. Totowa, NJ, USA: Humana Press.

Fisher, P. R., Noegel, A. A., Fechheimer, M., Rivero, F., Prassler, J. and Gerisch, G. (1997). Photosensory and thermosensory responses in Dictyostelium slugs are specifically impaired by absence of the F-actin cross-linking gelation factor (ABP-120). Curr. Biol. 7, 889-892.

Fox, J. W., Lamperti, E. D., Eksioglu, Y. Z., Hong, S. E., Feng, Y., Graham, D. A., Scheffer, I. E., Dobyns, W. B., Hirsch, B. A., Radtke, R. A. et al. (1998). Mutations in filamin 1 prevent migration of cerebral cortical neurons in human periventricular heterotopia. Neuron 21, 1315-1325.

Fucini, P., Koppel, B., Schleicher, M., Lustig, A., Holak, T. A., Muller, R., Stewart, M. and Noegel, A. A. (1999). Molecular architecture of the rod domain of the Dictyostelium gelation factor (ABP120). J. Mol. Biol. 291, 10171023

Fukuzawa, M., Abe, T. and Williams, J. (2003). The Dictyostelium prestalk cell inducer DIF regulates nuclear accumulation of a STAT protein by controlling its rate of export from the nucleus. Development 130, 797-804. 
Harwood, A. J. and Drury, L. (1990). New vectors for expression of the E. coli lacZ gene in Dictyostelium. Nucleic Acids Res. 18, 4292

Huang, E., Blagg, S. L., Keller, T., Katoh, M., Shaulsky, G. and Thompson, C. R. L. (2006). bZIP transcription factor interactions regulate DIF responses in Dictyostelium. Development 133, 449-458.

Huibregtse, J. M., Scheffner, M., Beaudenon, S. and Howley, P. M. (1995). A family of proteins structurally and functionally related to the E6-AP ubiquitinprotein ligase. Proc. Natl. Acad. Sci. USA 92, 5249

Jiang, Y., Levine, H. and Glazier, J. (1998). Possible cooperation of differential adhesion and chemotaxis in mound formation of Dictyostelium. Biophys. J. 75, 2615-2625

Katoh, M., Shaw, C., Xu, Q., Van Driessche, N., Morio, T., Kuwayama, H., Obara, S., Urushihara, H., Tanaka, Y. and Shaulsky, G. (2004). An orderly retreat: dedifferentiation is a regulated process. Proc. Natl. Acad. Sci. USA 101, 7005-7010.

Kay, R. R. (1998). The biosynthesis of differentiation-inducing factor, a chlorinated signal molecule regulating Dictyostelium development. J. Biol. Chem. 273, 26692675.

Kay, R. R. and Thompson, C. R. L. (2009). Forming patterns in development without morphogen gradients: scattered differentiation and sorting out. In Generation and Interpretation of Morphogen Gradients. Cold Spring Harb. Perspect. Biol. doi:10.1101/cshperspect.a001503.

Keller, T. and Thompson, C. R. (2008). Cell type specificity of a diffusible inducer is determined by a GATA family transcription factor. Development 135, 1635 1645.

Kessin, R. H. (2001). Dictyostelium. Cambridge, UK: Cambridge University Press.

Khaire, N., Muller, R., Blau-Wasser, R., Eichinger, L., Schleicher, M., Rief, M. Holak, T. A. and Noegel, A. A. (2007). Filamin-regulated F-actin assembly is essential for morphogenesis and controls phototaxis in Dictyostelium. J. Biol. Chem. 282, 1948-1955

Knuth, M., Khaire, N., Kuspa, A., Lu, S. J., Schleicher, M. and Noegel, A. A. (2004). A novel partner for Dictyostelium filamin is an alpha-helical developmentally regulated protein. J. Cell Sci. 117, 5013-5022.

Kurimoto, K., Yabuta, Y., Ohinata, Y., Ono, Y., Uno, K. D., Yamada, R. G., Ueda, H. R. and Saitou, M. (2006). An improved single-cell cDNA amplification method for efficient high-density oligonucleotide microarray analysis. Nucleic Acids Res. 34, e42

Lam, T. Y., Pickering, G., Geltosky, J. and Siu, C. H. (1981). Differential cell cohesiveness expressed by prespore and prestalk cells of Dictyostelium discoideum. Differentiation 20, 22-28

Maruo, T., Sakamoto, H., Iranfar, N., Fuller, D., Morio, T., Urushihara, H., Tanaka, Y., Maeda, M. and Loomis, W. F. (2004). Control of cell type proportioning in Dictyostelium discoideum by differentiation-inducing factor as determined by in situ hybridization. Eukaryot. Cell 3, 124-128.

Matsukuma, S. and Durston, A. J. (1979). Chemotactic cell sorting in Dictyostelium discoideum. J. Embryol. Exp. Morphol. 50, 243-251.

Morrison, A., Blanton, R. L., Grimson, M., Fuchs, M., Williams, K. and Williams, J. (1994). Disruption of the gene encoding the EcmA, extracellula matrix protein of Dictyostelium alters slug morphology. Dev. Biol. 163, 457-466.

Nagano, T., Yoneda, T., Hatanaka, Y., Kubota, C., Murakami, F. and Sato, M. (2002). Filamin A-interacting protein (FILIP) regulates cortical cell migration out of the ventricular zone. Nat. Cell Biol. 4, 495-501.

Nelson, M. K., Clark, A., Abe, T., Nomura, A., Yadava, N., Funair, C. J., Jermyn, K. A., Mohanty, S., Firtel, R. A. and Williams, J. G. (2000). An FBoxMD40 repeat-containing protein important for Dictyostelium cell-type proportioning, slug behaviour, and culmination. Dev. Biol. 224, 42-59.

Noegel, A. A., Rapp, S., Lottspeich, F., Schleicher, M. and Stewart, M. (1989). The Dictyostelium gelation factor shares a putative actin binding site with alphaactinin and dystrophin and also has a rod domain containing six 100-residue motifs that appear to have a cross-beta conformation. J. Cell Biol. 109, 607-618.

Ohta, Y., Suzuki, N., Nakamura, S., Hartwig, J. H. and Stossel, T. P. (1999). The small GTPase RalA targets filamin to induce filopodia. Proc. Natl. Acad. Sci. USA 96, 2122-2128

Onji, T., Takagi, M. and Shibata, N. (1987). Calpain abolishes the effect of filamin on the actomyosin system in platelets. Biochim. Biophys. Acta 912, 283-286.
Plusa, B., Piliszek, A., Frankenberg, S., Artus, J. and Hadjantonakis, A. K. (2008). Distinct sequential cell behaviours direct primitive endoderm formation in the mouse blastocyst. Development 135, 3081-3091.

Popowicz, G. M., Schleicher, M., Noegel, A. A. and Holak, T. A. (2006) Filamins: promiscuous organizers of the cytoskeleton. Trends Biochem. Sci. 31, 411-419.

Robertson, S. P. (2005). Filamin A: phenotypic diversity. Curr. Opin. Genet. Dev. 15, 301-307.

Saito, T., Kato, A. and Kay, R. R. (2008). DIF-1 induces the basal disc of the Dictyostelium fruiting body. Dev. Biol. 317, 444-453.

Schaefer, A. M., Hadwiger, G. D. and Nonet, M. L. (2000). rpm-1, a conserved neuronal gene that regulates targeting and synaptogenesis in $\mathrm{C}$. elegans. Neuron 26, 345-356.

Scheffner, M. and Staub, O. (2007). HECT E3s and human disease. BMC Biochem. 8 Suppl. 1, S6.

Scheffner, M., Nuber, U. and Huibregtse, J. M. (1995). Protein ubiquitination involving an E1-E2-E3 enzyme ubiquitin thioester cascade. Nature 373, 81-83.

Siegert, F. and Weijer, C. J. (1995). Spiral and concentric waves organize multicellular Dictyostelium mounds. Curr. Biol. 5, 937-943.

Siu, C. H., Harris, T. J., Wang, J. and Wong, E. (2004). Regulation of cell-cell adhesion during Dictyostelium development. Semin. Cell Dev. Biol. 15, 633-641.

Sussman, M. (1987). Cultivation and synchronous morphogenesis of Dictyostelium under controlled experimental conditions. Methods Cell Biol. 28 9-29.

Sussman, M., Schindler, J. and Kim, H. (1978). "Sluggers", a new class of morphogenetic mutants of D. discoideum. Exp. Cell Res. 116, 217-227.

Thompson, C. R. L. and Kay, R. R. (2000). The role of DIF-1 signaling in Dictyostelium development. Mol. Cell 6, 1509-1514.

Thompson, C. R. L., Fu, Q., Buhay, C., Kay, R. R. and Shaulsky, G. (2004a). A bZIP/bRLZ transcription factor required for DIF signaling in Dictyostelium. Development 131, 513-523.

Thompson, C. R. L., Reichelt, S. and Kay, R. R. (2004b). A demonstration of pattern formation without positional information in Dictyostelium. Dev. Growth Differ. 46, 363-369.

Townes, P. L. and Holtfreter, J. (1955). Directed movements and selective adhesion of embryonic amphibian cells. J. Exp. Zool. 128 53-120.

Traynor, D., Kessin, R. H. and Williams, J. G. (1992). Chemotactic sorting to CAMP in the multicellular stages of Dictyostelium development. Proc. Natl. Acad. Sci. USA 89, 8303-8307

Tu, Y., Wu, S., Shi, X., Chen, K. and Wu, C. (2003). Migfilin and Mig-2 link focal adhesions to filamin and the actin cytoskeleton and function in cell shape modulation. Cell 113, 37-47

Van Driessche, N., Shaw, C., Katoh, M., Morio, T., Sucgang, R., Ibarra, M., Kuwayama, H., Saito, T., Urushihara, H., Maeda, M. et al. (2002). A transcriptional profile of multicellular development in Dictyostelium discoideum. Development 129, 1543-1552.

Wan, H. I., DiAntonio, A., Fetter, R. D., Bergstrom, K., Strauss, R. and Goodman, C. S. (2000). Highwire regulates synaptic growth in Drosophila. Neuron 26, 313-329.

Wang, B. and Kuspa, A. (2002). CulB, a putative ubiquitin ligase subunit, regulates prestalk cell differentiation and morphogenesis in Dictyostelium spp. Eukaryot. Cell 1, 126-136.

Whitney, N., Pearson, L. J., Lunsford, R., McGill, L., Gomer, R. H. and Lindsey, D. F. (2006). A putative Ariadne-like ubiquitin ligase is required for Dictyostelium discoideum development. Eukaryot. Cell 5, 1820-1825.

Williams, J. G. (2006). Transcriptional regulation of Dictyostelium pattern formation. EMBO Rep. 7, 694-698.

Williams, J. G., Duffy, K. T., Lane, D. P., Mcrobbie, S. J., Harwood, A. J., Traynor, D., Kay, R. R. and Jermyn, K. A. (1989a). Origins of the prestalkprespore pattern in Dictyostelium development. Cell 59, 1157-1163.

Williams, J. G., Jermyn, K. A. and Duffy, K. T. (1989b). Formation and anatomy of the prestalk zone of Dictyostelium. Development 107 Suppl., 91-97.

Zhukovskaya, N., Fukuzawa, M., Yamada, Y., Araki, T. and Williams, J. G. (2006). The Dictyostelium bZIP transcription factor DimB regulates prestalkspecific gene expression. Development 133, 439-448. 